\title{
Getting our Chemicals Legislation Working Better Across the Board
}

The primary objective of ECHA is to protect the environment and health. Alongside the reduction of animal testing, we also work to ensure the free circulation of substances on the market and aim to foster innovation and enhance competitiveness. This work, in turn, helps European companies improve their competitiveness both externally and internally.

It is not an easy task to draw out the matrix of chemicals legislation that ECHA is part of. But let me offer you one representation of it. On one hand, there are many pieces of product legislation with an interface for chemicals - for example, those addressing toys, biocides, plant protection products, food additives and food contact materials. On the other hand, there are a number of pieces of legislation that protect certain populations - the most prominent for ECHA's daily work being the legislation that protects workers. The European Commission's environment policy contains various compartments, for example, the Water Framework Directive, which is closely related to ECHA's work.

So, how do the different pieces of legislation ECHA manages fit into the EU legislative framework for chemicals and its implementation? If we start from the core, REACH works as the data generator and regulatory gap filler whereas CLP is the harmonised approach which looks into reducing hazard. Both of them prominently contribute to the whole framework of chemicals legislation as a large majority of the chemicals on the EU market rely on the data as well as the hazard identification and assessment coming from REACH and CLP. And REACH requires industry to document in their registration dossiers how they implement this whole framework for each chemical on the EU market.

\section{Cross-Legislative and Cross-Agency Interfaces}

In addition to interfaces between different pieces of chemicals legislation, there are also those between different actors and different regulatory agencies. There are many cases where substances and processes are looked at from different points of view.

For example, many food additives are being registered under REACH which means that they need to be aligned with REACH information requirements. But they also need to align with requirements under food additives legislation. 
Silver is being assessed under REACH, but also under the Biocidal Products Regulation - both regulations are managed by ECHA. Chromium trioxide is on the REACH Authorisation List so its use requires authorisation but there is also a clear interface with worker protection legislation managed by other authorities. Under REACH, CLP and the Biocidal Products Regulation, work is ongoing on endocrine disruptors where we systematically try to identify and assess substances with these properties. These substances may also end up being used in cosmetics, which are regulated under a separate piece of legislation.

Phthalates are a good example when discussing interfaces between agencies. When we did our assessment on phthalates, we needed to exclude exposure through food contact materials because our opinion was used to restrict the use of phthalates in consumer plastics and some other consumer goods. However, the focus of the assessment carried out by the European Food Safety Authority (EFSA) is exactly on the food contact material. Therefore, it is important that we identify areas where we can cooperate at an early point and make sure we are using the same basis for our assessments.

The interfaces work well in many cases, but we can certainly still improve. We can further harmonise our approaches, increase the information flow between different actors and improve efficiencies and consistency. As said, we need to use the same information and clarify where the biggest differences are in our approaches.

\section{The Circular Economy}

One of the biggest ongoing policy discussions concerns the circular economy and we at ECHA are closely following the policy development taking place in Brussels. Materials that should be recycled a number of times - ideally, an infinite amount of times - are at the core of a circular economy. But if we look at circularity from a materials perspective, we need to be able to define what a material is. In the language of chemistry, a material is nothing but a mixture. For us, this makes it all much easier, because we know and understand how to deal with mixtures. So, if we continue this analogy and say that whatever comes out of the waste stream is a mixture, then the issue of what is in the mixture is very similar to when mixtures are imported into the EU. And when companies import mixtures from outside Europe, the substances included in those mixtures must be registered under REACH.

Another part of the discussion is the realisation that in the future more and more substances that need to be registered will no longer be virgin materials but will be coming in through the waste stage. This makes it all the more important that we are able to properly track and trace these chemicals. Therefore, I am very happy that legislators have given us the task of setting up a waste database for this purpose.

We have a very good basis to start looking at chemicals within a circular economy and we can start contributing as soon as policy makers ask for support from the scientific technical bodies. 


\section{REACH as a Cornerstone}

As we have seen, the interfaces need to be further improved and the information base strengthened. And this leads us to the discussion on REACH compliance. It is being taken very seriously - and so it should, because if the registered substances are non-compliant, REACH cannot work as planned. And in turn, none of the other pieces of legislation that use REACH data, can work as they should. So REACH is a cornerstone in getting our implementation on chemicals legislation working better across the board.

Another important aspect to mention is REACH authorisation, which is a very strong driver for substitution. Therefore, the better we manage the authorisation process as a whole, the better the substitution mechanism will work. This innovation will contribute to building an EU chemicals market that will be fit for an economy that relies on circularity. 